\title{
Current State of Nanoemulsions in Drug Delivery
}

\author{
Charles Lovelyn, Anthony A. Attama*
}

Department of Pharmaceutics, University of Nigeria, Nsukka, Nigeria.

E-mail: ${ }^{*}$ anthony.attama@unn.edu.ng, ${ }^{*}$ aaattama@yahoo.com

Received September $20^{\text {th }}, 2011$; revised October $27^{\text {th }}, 2011$; accepted November $14^{\text {th }}, 2011$.

\begin{abstract}
Nanoemulsions have attracted great attention in research, dosage form design and pharmacotherapy. This is as a result of a number of attributes peculiar to nanoemulsions such as optical clarity, ease of preparation, thermodynamic stability and increased surface area. Nanoemulsions also known as submicron emulsions serve as vehicles for the delivery of active pharmaceutical ingredients as well as other bioactives. They are designed to address some of the problems associated with conventional drug delivery systems such as low bioavailability and noncompliance. The importance of design and development of emulsion nanocarrier systems aimed at controlling and/or improving required bioavailability levels of therapeutic agents cannot be overemphasized. Reducing droplet sizes to the nanoscale leads to some very interesting physical properties, such as optical transparency and unusual elastic behaviour. This review sheds light on the current state of nanoemulsions in the delivery of drugs and other bioactives. The morphology, formulation, characteristics and characterization of nanoemulsions were also addressed.
\end{abstract}

Keywords: Nanoemulsion, Preparation, Characterization, Application in Drug Delivery, Patents

\section{Introduction}

Nanoemulsions are oil-in-water $(\mathrm{o} / \mathrm{w})$ emulsions with mean droplet diameters ranging from 50 to $1000 \mathrm{~nm}$. Usually, the average droplet size is between 100 and 500 $\mathrm{nm}$. The particles can exist as oil-in-water and waterin-oil forms, where the core of the particle is either oil or water, respectively. Nanoemulsions are made from surfactants approved for human consumption and common food substances that are "Generally Recognized as Safe" (GRAS) by the FDA. These emulsions are easily produced in large quantities by mixing a water-immiscible oil phase with an aqueous phase under high shear stress, or mechanical extrusion process that is available worldwide [1].

Nanoemulsions are also referred to as miniemulsions, ultrafine emulsions and submicron emulsions. Phase behaviour studies have shown that the size of the droplets is governed by the surfactant phase structure (bicontinuous microemulsion or lamellar) at the inversion point induced by either temperature or composition.

The capacity of nanoemulsions to dissolve large quantities of hydrophobics, along with their mutual compatibility and ability to protect the drugs from hydrolysis and enzymatic degradation make them ideal vehicles for the purpose of parenteral transport. Further, the frequency and dosage of injections can be reduced throughout the drug therapy period as these emulsions guarantee the release of drugs in a sustained and controlled mode over long periods of time. Additionally, the lack of flocculation, sedimentation and creaming, combined with a large surface area and free energy, offer obvious advantages over emulsions of larger particle size, for this route of administration. Their very large interfacial area positively influences the drug transport and their delivery, along with targeting them to specific sites [2,3].

Reducing droplet sizes to the nanoscale leads to some very interesting physical properties, such as optical transparency and unusual elastic behaviour. In the world of nanomaterials, nanoemulsions hold great promise as useful dispersions of deformable nanoscale droplets that can have flow properties ranging from liquid to highly solid and optical properties ranging from opaque to nearly transparent. Moreover, it is very likely that nanoemulsions will play an increasingly important role commercially, since they can typically be formulated using significantly less surfactant than is required for nanostructured lyotropic microemulsion phases. Nanoemulsions are part of a broad class of multiphase colloidal dispersions. Although some lyotropic liquid crystalline phases, also known as "micellar phases", "mesophases", and "microemulsions", may appear to be similar to nanoemulsions in composition and nanoscale structure, 
such phases are actually quite different [4]. Lyotropic liquid crystals are equilibrium structures comprised of liquids and surfactant, such as lamellar sheets, hexagonally packed columns, and wormlike micellar phases, that form spontaneously through thermodynamic self assembly. By contrast, nanoemulsions do not form spontaneously; an external shear must be applied to rupture larger droplets into smaller ones. Compared to microemulsion phases, relatively little is known about creating and controlling nanoemulsions. This is primarily because extreme shear, well beyond the reach of ordinary mixing devices, must be applied to overcome the effects of surface tension to rupture the droplets into the nanoscale regime [4].

\section{Preparation of Nanoemulsions}

Nanoemulsions are non-equilibrium systems of structured liquids [2-4], and so their preparation involves the input of a large amount of either energy or surfactants and in some cases a combination of both. As a result, high energy or low energy methods can be used in their formulation [3]. The high-energy method utilizes mechanical devices to create intensely disruptive forces which break up the oil and water phases to form nanosized droplets. This can be achieved with ultrasonicators, microfluidiser and high pressure homogenisers [4-6]. Particle size here will depend on the type of instruments employed and their operating conditions like time and temperature along with sample properties and composition [7]. This method allows for a greater control of particle size and a large choice of composition, which in turn controls the stability, rheology and colour of the emulsion. Although high-energy emulsification methods yield nanoemulsions with desired properties and have industrial scalability, they may not be suitable for thermolabile drugs such as retinoids and macromolecules, including proteins, enzymes and nucleic acids.

Nanoemulsion can be prepared by a low-energy emulsification method, which has been recently developed according to the phase behavior and properties of the constituents, to promote the formation of ultra-small droplets $[8,9]$. These low-energy techniques include selfemulsification, phase transition and phase inversion temperature methods [10]. The low energy method is interesting because it utilizes the stored energy of the system to form small droplets. This emulsification can be brought about by changing the parameters which would affect the hydrophilic lipophilic balance (HLB) of the system like temperature, composition, etc. $[11,12]$.

Energy is usually required in emulsion formulation because the process may be non-spontaneous. The production of nanoemulsions costs more energy than that required to produce macroemulsions. Presence of surfac- tants help lower the surface tensions between oil and water. Small molecules such as non-ionic surfactants lower surface tension more than polymeric surfactants such as poly(vinyl alcohol). Another important role of the surfactant is its effect on the interfacial dilatational modulus [13]. During emulsification an increase in the interfacial area takes place and this causes a reduction in surface excess. The equilibrium is restored by adsorption of surfactant from the bulk, but this takes time (shorter times occur at higher surfactant activity). Because of the lack or slowness of equilibrium with polymeric surfactants, dilatational modulus will not be the same for expansion and compression of the interface [13]. In practice, surfactant mixtures are used and these have pronounced effects on surface tension and dilatational modulus. Some specific surfactant mixtures give lower surface tension values than either of the two individual components. Polymer-surfactant mixtures may show some synergistic surface activity.

Another important role of the emulsifier is to prevent shear-induced coalescence during emulsification. The requirement is that the continuous phase has a significant excess of surfactant. This excess enables new surface area of the nanoscale droplets to be rapidly coated during emulsification, thereby inhibiting shear-induced coalescence. This excess is generally in the form of surfactant micelles in the continuous phase. These micelles dissociate into monomers that rapidly adsorb onto the surfaces of newly created droplets [4].

\subsection{Methods of Preparation of Nanoemulsions}

\subsubsection{High Pressure Homogenization}

This technique makes use of high-pressure homogenizer/ piston homogenizer to produce nanoemulsions of extremely low particle size (up to $1 \mathrm{~nm}$ ). During this process, several forces, such as hydraulic shear, intense turbulence and cavitation, act together to yield nanoemulsions with extremely small droplet size. The resultant product can be re-subjected to high-pressure homogenization until nanoemulsion with desired droplet size and polydispersity index is obtained. The production of small droplets (submicron) requires application of high energy. Several procedures may be applied to enhance the efficiency of emulsification when producing nanoemulsions. The emulsion is preferably prepared at high volume faction of the disperse phase and diluted afterwards. However, very high phase volume ratios may result in coalescence during emulsification, but more surfactant could be added to create a smaller reduction in effective surface tension and possibly diminishing recoalescence. Surfactant mixtures that show more reduction in surface tension than the individual components could also be used. If possible the surfactant is dissolved in the disperse phase 
rather than the continuous phase; this often leads to smaller droplets. It may be useful to emulsify in steps of increasing intensity, particularly with emulsions having highly viscous disperse phase.

\subsubsection{Microfluidization}

Microfluidization is a patented mixing technology, which makes use of a device called microfluidizer. This device uses a high-pressure positive displacement pump (500 $20,000 \mathrm{psi}$ ), which forces the product through the interaction chamber, consisting of small channels called "microchannels". The product flows through the microchannels on to an impingement area resulting in very fine particles of submicron range. The two solutions (aqueous phase and oily phase) are combined together and processed in an inline homogenizer to yield a coarse emulsion [1]. The coarse emulsion is introduced into a microfluidizer where it is further processed to obtain a stable nanoemulsion. The coarse emulsion is passed through the interaction chamber of the microfluidizer repeatedly until the desired particle size is obtained. The bulk emulsion is then filtered through a filter under nitrogen to remove large droplets resulting in a uniform nanoemulsion. Highpressure homogenization and microfluidization can be used for fabrication of nanoemulsions at laboratory and industrial scale, whereas ultrasonic emulsification is mainly used at laboratory scale.

\subsubsection{Phase Inversion Temperature Technique}

Studies on nanoemulsion formulation by the phase inversion temperature method have shown a relationship between minimum droplet size and complete solubilization of the oil in a microemulsion bicontinuous phase independently of whether the initial phase equilibrium is single or multiphase. Due to their small droplet size nanoemulsions possess stability against sedimentation or creaming with Ostwald ripening forming the main mechanism of nanoemulsion breakdown [9]. Phase inversion in emulsions can be one of two types: transitional inversion induced by changing factors which affect the HLB of the system, e.g. temperature and/or electrolyte concentration, and catastrophic inversion, which can also be induced by changing the HLB number of the surfactant at constant temperature using surfactant mixtures [13].

Phase inversion temperature (PIT) method employs temperature-dependent solubility of nonionic surfactants, such as polyethoxylated surfactants, to modify their affinities for water and oil as a function of the temperature. It has been observed that polyethoxylated surfactants tend to become lipophilic on heating owing to dehydration of polyoxyethylene groups. This phenomenon forms a basis of nanoemulsion fabrication using the PIT method. In the PIT method, oil, water and nonionic surfactants are mixed together at room temperature. This mixture typically comprises o/w microemulsions coexisting with excess oil, and the surfactant monolayer exhibits positive curvature. When this macroemulsion is heated gradually, the polyethoxylated surfactant becomes lipophilic and at higher temperatures, the surfactant gets completely solubilized in the oily phase and the initial $\mathrm{o} / \mathrm{w}$ emulsion undergoes phase inversion to $\mathrm{w} / \mathrm{o}$ emulsion. The surfactant monolayer has negative curvature at this stage. This method involves heating of the components and it may be difficult to incorporate thermolabile drugs, such as tretinoin and peptides, without affecting their stability. Although it may be possible to reduce the PIT of the dispersion using a mixture of components (surfactants) with suitable characteristics, in order to minimize degradation of thermolabile drugs.

\subsubsection{Solvent Displacement Method}

The solvent displacement method for spontaneous fabrication of nanoemulsion has been adopted from the nanoprecipitation method used for polymeric nanoparticles. In this method, oily phase is dissolved in water-miscible organic solvents, such as acetone, ethanol and ethyl methyl ketone. The organic phase is poured into an aqueous phase containing surfactant to yield spontaneous nanoemulsion by rapid diffusion of organic solvent. The organic solvent is removed from the nanoemulsion by a suitable means, such as vacuum evaporation. Spontaneous nanoemulsification has also been reported when solution of organic solvents containing a small percentage of oil is poured into aqueous phase without any surfactant.

Solvent displacement methods can yield nanoemulsions at room temperature and require simple stirring for the fabrication. Hence, researchers in pharmaceutical sciences are employing this technique for fabricating nanoemulsions mainly for parenteral use. However, the major drawback of this method is the use of organic solvents, such as acetone, which require additional inputs for their removal from nanoemulsion. Furthermore, a high ratio of solvent to oil is required to obtain a nanoemulsion with a desirable droplet size. This may be a limiting factor in certain cases. In addition, the process of solvent removal may appear simple at laboratory scale but can pose several difficulties during scale-up.

\subsubsection{Phase Inversion Composition Method (Self-Nanoemulsification Method)}

This method has drawn a great deal of attention from scientists in various fields (including pharmaceutical sciences) as it generates nanoemulsions at room temperature without use of any organic solvent and heat. Kinetically stable nanoemulsions with small droplet size $(\sim 50 \mathrm{~nm})$ can be generated by the stepwise addition of water into solution of surfactant in oil, with gentle stir- 
ring and at constant temperature. The spontaneous nanoemulsification has been related to the phase transitions during the emulsification process and involves lamellar liquid crystalline phases or D-type bicontinuous microemulsion during the process. Nanoemulsions obtained from the spontaneous nanoemulsification process are not thermodynamically stable, although they might have high kinetic energy and long-term colloidal stability.

\subsection{Advantages of Nanoemulsions as Drug Delivery Systems}

The attraction of nanoemulsions for application in personal care and cosmetics as well as in health care is due to the following advantages $[13,14]$ :

1) The very small droplet size causes a large reduction in the gravity force and the Brownian motion may be sufficient for overcoming gravity. This means that no creaming or sedimentation occurs on storage.

2) The small droplet size also prevents any flocculation of the droplets. Weak flocculation is prevented and this enables the system to remain dispersed with no separation.

3) The small droplets also prevent their coalescence, since these droplets are elastic, surface fluctuations are prevented.

4) Nanoemulsions are suitable for efficient delivery of active ingredients through the skin. The large surface area of the emulsion system allows rapid penetration of actives.

5) The transparent nature of the system, their fluidity (at reasonable oil concentrations) as well as the absence of any thickeners may give them a pleasant aesthetic character and skin feel.

6) Unlike microemulsions (which require a high surfactant concentration, usually in the region of $20 \%$ and higher), nanoemulsions can be prepared using reasonable surfactant concentration. For a $20 \%$ o/w nanoemulsion, a surfactant concentration in the region of $5 \%-10 \%$ may be sufficient. Nanoemulsions are usually formulated with surfactants, which are approved for human consumption (GRAS), they can be taken by enteric route.

7) The small size of the droplets allows them to deposit uniformly on substrates. Wetting, spreading and penetration may be also enhanced as a result of the low surface tension of the whole system and the low interfacial tension of the $\mathrm{o} / \mathrm{w}$ droplets.

8) Nanoemulsions can be applied for delivery of fragrants, which may be incorporated in many personal care products. This could also be applied in perfumes, which are desirable to be formulated alcohol free.

9) Nanoemulsions may be applied as a substitute for liposomes and vesicles (which are much less stable) and it is possible in some cases to build lamellar liquid crys- talline phases around the nanoemulsion droplets.

\subsection{Disadvantages of Nanoemulsion Drug Delivery Systems}

Inspite of the above advantages, nanoemulsions have only attracted interest in recent years for the following reasons $[13,14]$ :

1) Preparation of nanoemulsions requires in many cases special application techniques, such as the use of high pressure homogenisers as well as ultrasonics. Such equipment (such as the Microfluidiser) became available only in recent years.

2) There is a perception in the personal care and cosmetic industry that nanoemulsions are expensive to produce. Expensive equipment are required as well as the use of high concentrations of emulsifiers.

3) Lack of understanding of the mechanism of production of submicron droplets and the role of surfactants and cosurfactants.

4) Lack of demonstration of the benefits that can be obtained from using nanoemulsions when compared with the classical macroemulsion systems.

5) Lack of understanding of the interfacial chemistry that is involved in production of nanoemulsions.

\section{Applications of Nanoemulsions in Drug Delivery}

\subsection{Nanoemulsions and Intranasal Drug Delivery}

Intranasal drug delivery system has now been recognized as a reliable route for the administration of drugs next to parenteral and oral routes. Nasal mucosa has emerged as a therapeutically viable channel for the administration of systemic drugs and also appears to be a favourable way to overcome the obstacles for the direct entry of drugs to the target site [15]. This route is also painless, non-invasive and well tolerated. The nasal cavity is one of the most efficient sites because of its reduced enzymatic activity, high availability of immunoactive sites and its moderately permeable epithelium [16]. There are several problems associated with targeting drugs to brain, especially the hydrophilic ones and those of high molecular weight. This is because of the impervious nature of the endothelium, which divides the systemic circulation and barrier between the blood and brain [17]. The olfactory region of the nasal mucosa provides a direct connection between the nose and brain, and by the use of nanoemulsions loaded with drugs, conditions such as Alzheimer's disease, migraine, depression, schizophrenia, Parkinson's diseases, meningitis, etc. can be treated $[18,19]$.

Preparation of nanoemulsions containing risperidone for its delivery to the brain via nose has been reported [19]. It is inferred that this emulsion is more effective 
through the nasal rather than intravenous route. Another application of intranasal drug delivery system in therapeutics is their use in development of vaccines. Immunity is achieved by the administration of mucosal antigen. Currently, the first intranasal vaccine has been marketed [20]. Among the possible delivery systems, the use of nano based carriers hold a great promise to protect the biomolecules, promote nanocarrier interaction with mucosae and to direct antigen to the lymphoid tissues. Therefore the use of nanoemulsions in intranasal drug delivery system is set to bring about significant results in targeting drugs to the brain in treatment of diseases related to the central nervous system [21].

\subsection{Nanoemulsions and Transdermal Delivery}

Drug delivery through the skin to the systemic circulation is convenient for a number of clinical conditions due to which there has been a considerable interest in this area $[22,23]$. It offers the advantage of steady state controlled drug delivery over extended period of time, with self administration also being possible, which may not be the case with parenteral route. The drug input can be eliminated at any time by the patient just by removing the transdermal patch. Their transparent nature and fluidity, confers on nanoemulsions a pleasant skin feel. An extra advantage is the total absence of gastrointestinal side effects like irritation and bowel ulcers which are invariably associated with oral delivery. Transdermal drug products have been developed for a number of diseases and disorders including cardiovascular conditions, Parkinsons' and Alzheimer diseases, anxiety, depression, etc. However, the fundamental disadvantage which limits the use of this mode of administration is the barrier imposed by the skin for effective penetration of the bioactives. The three routes by which drugs can primarily penetrate the skin are through the hair follicles, sweat ducts or directly across stratum corneum, which restricts their absorption to a large extent and limits their bioavailability. For improved drug pharmacokinetics and targeting, the primary skin barriers need to be overcome. Also the locally applied drug redistribution through cutaneous blood and lymph vessel system needs to be controlled. Nano sized emulsions are able to easily penetrate the pores of the skin and reach the systemic circulation thus getting channelized for effective delivery [2]. Caffeine has been used for treatment of different types of cancer by oral delivery. Water-in-oil nanoemulsion formulations of caffeine have been developed for transdermal drug delivery. Comparison of in vitro skin permeation profile between these and aqueous caffeine solutions showed significant increase in permeability parameters for the nanoemulsion loaded drugs [24].

Use of nanoemulsions in transdermal drug delivery represents an important area of research in drug delivery, which enhances the therapeutic efficacy and also the bioavailability of the drugs without any adverse effects. It is also regarded as a promising technique with many advantages including, high storage stability, low preparation cost, thermodynamic stability, absence of organic solvents, and good production feasibility. They have also made the plasma concentration profiles and bioavailability of drugs reproducible. These systems are being used currently to provide dermal and surface effects, and for deeper skin penetration [2]. Many studies have shown that nanoemulsion formulations possess improved transdermal and dermal delivery properties in vitro [25-33], as well as in vivo [34-36]. Nanoemulsions have improved transdermal permeation of many drugs over the conventional topical formulations such as emulsions [37,38] and gels $[39,40]$.

\subsection{Nanoemulsions and Parenteral Drug Delivery}

This is one of the most common and effective routes of drug administration usually adopted for actives with low bioavailability and narrow therapeutic index. Their capacity to dissolve large quantities of hydrophobics, together with their mutual compatibility and ability to protect the drugs from hydrolysis and enzymatic degradation make nanoemulsions ideal vehicles for the purpose of parenteral transport. Further, the frequency and dosage of injections can be reduced throughout the drug therapy period as these emulsions guarantee the release of drugs in a sustained and controlled mode over long periods of time. Additionally, the lack of flocculation, sedimentation and creaming, combined with a large surface area and free energy, offer obvious advantages over emulsions of larger particle size, for this route of administration [2]. Their very large interfacial area positively influences the drug transport and their delivery, along with targeting them to specific sites. Major clinical and preclinical trials have hence been carried out with parenteral nanoemulsion based carriers. The advances in these novel drug delivery systems have been reviewed by Patel and Patel [41]. Nanoemulsions loaded with thalidomide have been synthesized where a dose as low as $25 \mathrm{mg}$ leads to plasma concentrations which can be therapeutic [42]. However, a significant decrease in the drug content of the nanoemulsion was observed at $0.01 \%$ drug formulation after two months storage which could be overcome by the addition of polysorbate 80 . Chlorambucil, a lipophilic anticancer agent has been used against breast and ovarian cancer. Its pharmacokinetics and anticancer activity has been studied by loading it in parenteral emulsions prepared by high energy ultrasonication method. Treatment of colon adenocarcinoma in the mouse with 
this nanoemulsion leads to higher tumor suppression rate compared to plain drug solution treatment concluding that the drug loaded emulsion could be an effective carrier for its delivery in cancer treatment [43]. Carbamazepine, a widely used anticonvulsant drug had no parenteral treatment available for patients due to its poor water solubility. Kelmann et al. [44] have developed a nanoemulsion for its intravenous delivery, which showed favorable in vitro release kinetics.

\subsection{Nanoemulsions and Drug Targeting}

Another interesting application, which is experiencing an active development, is the use of nanoemulsion formulations, for controlled drug delivery and targeting [9]. Because of their submicron size, they can easily be targeted to the tumor area. Although nanoemulsions are chiefly seen as vehicles for administering aqueous insoluble drugs, they have more recently received increasing attention as colloidal carriers for targeted delivery of various anticancer drugs, photosensitizers, neutron capture therapy agents, or diagnostic agents. The development of magnetic nanoemulsions is an innovative approach for cancer therapy. These can deliver photosensitizers like Foscan ${ }^{\circledR}$ to deep tissue layers across the skin thereby inducing hyperthermia for subsequent free radical generation. This methodology can be used for the treatment of cancer in the form of photodynamic therapy [45].

\subsection{Nanoemulsions and Vaccine Delivery}

A vaccine carrier system using nanoemulsions is currently being researched. This medication delivery system uses nanotechnology to vaccinate against human immunodeficiency virus (HIV). There is recent evidence that HIV can infect the mucosal immune system. Therefore, developing mucosal immunity through the use of nanoemulsions may become very important in the future fight against HIV [46]. The oil-based emulsion is administered in the nose, as opposed to traditional vaccine routes. Research is demonstrating that genital mucosa immunity may be attained with vaccines that are administered into the nasal mucosa [47].

\subsection{Nanoemulsions and Pulmonary Drug Delivery}

Until now, the submicron emulsion system has not yet been fully exploited for pulmonary drug delivery and very little has been published in this area [48]. Emulsion systems have been introduced as alternative gene transfer vectors to liposomes [49]. Other emulsion studies for gene delivery (non-pulmonary route) have shown that binding of the emulsion/DNA complex was stronger than liposomal carriers [50]. This stable emulsion system delivered genes more efficiently than liposomes [51]. Bivas-Benita et al. [52] reported that cationic submicron emulsions are promising carriers for DNA vaccines to the lung since they are able to transfect pulmonary epithelial cells, which possibly induce cross priming of antigen-presenting cells and directly activate dendritic cells, resulting in stimulation of antigen-specific T-cells. Therefore the nebulization of submicron emulsions will be a new and upcoming research area. However, extensive studies are required for the successful formulation of inhalable submicron emulsions due to possible adverse effects of surfactants and oils on lung alveoli function (adverse interactions with lung surfactant).

\subsection{Prophylactic in Bio-Terrorism Attack}

Based on their antimicrobial activity, research has begun on use of nanoemulsion as a prophylactic medication, a human protective treatment, to treat people exposed to bio-attack pathogens such as anthrax and ebola. A broad spectrum nanoemulsion was tested on surfaces by the USA army in Dec 1999 for decontamination of Anthrax spore surrogates. It was tested again by RestOps in March 2001 as a chemical decontamination agent. All tests were successful. The technology has been tested on gangrene and Clostridium botulism spores and can even be used on contaminated wounds to salvage limbs. The nanoemulsion technology can be formulated into a foam, liquid, cream, or spray to decontaminate a variety of materials as has been done by NanoBio Corporation [53].

\section{Characterization of Nanoemulsion}

Optical microscopy, even using differential interference contrast or other phase contrast methods, is generally not a viable method for examining nanoemulsions. As discussed below, more sophisticated techniques, such as dynamic light scattering, x-ray or neutron scattering, atomic force microscopy, or cryo-electron microscopy are typically required to explore the structure and behaviour of nanoemulsions [4].

Nanoemulsions have some interesting physical properties that distinguish them from ordinary microscale emulsions. For instance, microscale emulsions typically exhibit strong multiple scattering of visible light, and, as a result, have a white appearance. The multiple scattering occurs as the light is refracted many times through droplets, films, and plateau borders, provided there is a significant refractive index contrast between the dispersed and continuous phases. In the absence of optical absorption, photons that enter the emulsion are scattered many times by microscale structures before they leave the emulsion [4]. By contrast, the structures in nanoemulsions are much smaller than visible wavelengths, so most 
nanoemulsions appear optically transparent, even at large phase volume ratio and for large difference in refractive index. Nanoemulsions may loose their transparency with time as a result of increase in droplet size. Nanoemulsions have a much larger surface area to volume ratio than ordinary emulsions, so phenomena related to deformation of the droplets, such as the elastic modulus, are typically larger for nanoemulsions than ordinary emulsions. Due to the large surface to volume ratio of droplet interfaces in nanoemulsions, the concentration of surface- tant required to stabilize them is larger than for microscale emulsions, yet it is generally smaller than for lyotropic microemulsion phases. Different characterization parameters for nanoemulsions are discussed in the following sections:

\subsection{Morphology of Nanoemulsions}

The morphology of nanoemulsions can be determined by transmission electron microscopy (TEM) and scanning electron microscopy (SEM). SEM gives a three-dimensional image of the globules [54]. The samples are examined at suitable accelerating voltage, usually $20 \mathrm{kV}$, at different magnifications. A good analysis of surface morphology of disperse phase in the formulation is obtained through SEM. Image analysis software, (e.g., Leica Imaging systems, Cambridge, UK), may be employed to obtain an automatic analysis result of the shape and surface morphology [55].

In TEM, higher resolution images of the disperse phase are obtained. The sample is negatively stained with $1 \%$ aqueous solution of phosphotungstic acid or by dropping $2 \%$ uranyl acetate solution onto a $200 \mu \mathrm{m}$ mesh size Pioloform ${ }^{\text {TM}}$-coated copper grid or a microscopic carbon-coated grid using a micropipette and the sample examined under the transmission electron microscope (for e.g., Joel 1230, Tokyo, Japan) at $80 \mathrm{kV}$ [56]. Qualitative measurements of sizes and size distribution of TEM micrographs can be performed using a digital image processing programme [56].

\subsection{Nanoemulsion Droplet Size, Polydispersity and Zeta Potential}

Dynamic light scattering which is referred to as Photon Correlation Spectroscopy (PCS) is used to analyze the fluctuations in the intensity of scattering by droplets/ particles due to Brownian motion [57]. Nanoemulsion droplet size, polydispersity and zeta potential can be assessed by PCS using a particle size analyzer. This instrument also measures polydispersity index, which is a measure of the broadness of the size distribution derived from the cumulative analysis of dynamic light scattering. The polydispersity index indicates the quality or homogeneity of the dispersion [58]. PCS gives z-average par- ticle diameter. Laser diffraction is another technique for measuring particle size. The fundamental particle size distribution derived by this technique is volume based and is expressed in terms of the volume of equivalent spheres $\left(D_{N \%}\right)$ and weighted mean of the volume distribution (mass mean diameter). Since the laser diffraction system is used for this analysis, a rough equivalent of particle polydispersity could be given by two factors/ values namely, uniformity (how symmetrical the distribution is around the median point) and span (the width of the distribution). The span value is defined by the expression:

$$
\text { Span }=\left(D_{90 \%}-D_{10 \%}\right) / D_{50 \%}
$$

where $D_{N \%}(N=10 \%, 50 \%, 90 \%)$, means that the volume percentage of particles with diameters up to $D_{N \%}$ equals to $N \%$. The smaller the span value the narrower the particle size distribution.

\subsection{Viscosity Determination}

This is carried out using a viscometer. The viscosity of nanoemulsions is a function of the surfactant, water and oil components and their concentrations. Increasing the water content lowers the viscosity, while decreasing the amount of surfactant and cosurfactant increases interfacial tension between water and oil resulting in increased viscosity. Viscosity is very important for stability and efficient drug release. Nanoemulsion carrier formulations are basically oil-in-water and so in addition to being less greasy than water-in-oil formulations, often possess lower apparent viscosities. They are therefore expected to exhibit faster release of active ingredients and wash out easily after application on the skin surface. Various equipment and methods are available for assessment of rheological properties of nanoemulsion carriers. Monitoring of viscosity change is a method of assessing stability of liquid and semi-solid preparations including nanoemulsion formulations.

\subsection{In Vitro Skin Permeation Studies}

Franz diffusion cell is used to obtain the drug release profile of the nanoemulsion formulation in the case of formulations for transdermal application. The extent or depth of skin penetration by the released content can be visualized by confocal scanning laser microscopy. In vitro drug release can be determined by dispersing an amount of the preparation in the donor compartment of a Franz cell having a membrane as barrier and monitoring the appearance of the encapsulated drug in the reception medium, usually PBS ( $\mathrm{pH} 7.4)$ and stirring on a magnetic stirrer at $100 \mathrm{rpm}$ at $37^{\circ} \mathrm{C} \pm 1^{\circ} \mathrm{C}$. Samples $(1 \mathrm{ml})$ of the dispersion are withdrawn from the medium and replaced with an equivalent amount of the medium at definite in- 
tervals. The withdrawn sample is then filtered using a 0.22 - $50 \mu \mathrm{m}$ filter (e.g., Millipore, USA) and the drug released then analyzed using UV-visible spectroscopy at wavelength of peak absorption of the drug [59]. An alternative and popular method of ex-vivo release study is performed using diffusion cell. The skin is cut from the ear or abdomen and underlying cartilage and fats carefully removed. Appropriate size of skin is cut and placed on the diffusion cell which had earlier been filled with receptor solution. Samples of the vesicular preparation are then applied on the dorsal surface of the skin and the instrument started. At intervals, up to $24 \mathrm{~h}$, samples are withdrawn from the receptor medium and replaced with equal amounts of the medium and the withdrawn samples analyzed for the drug permeated using HPLC $[60,61]$ or UV spectroscopy. Semi-permeable membrane such as regenerated cellulose could be used in place of skin for in vitro release studies $[62,63]$. The flux $J$, of the drug across the skin or membrane is calculated from the formula:

$$
J=D \mathrm{~d} c / \mathrm{d} x
$$

where $D$ is the diffusion coefficient and is a function of the size, shape and flexibility of the diffusing molecule as well as the membrane resistance, $c$ is the concentration of the diffusing species, $x$ is the spatial coordinate [63].

In vivo release study otherwise referred to as dermatopharmacokinetics, is carried out by applying or administering the preparation to whole live animal. Blood samples are then withdrawn at intervals, centrifuged and the plasma analyzed for the drug content using HPLC. Results obtained from in vitro and in vivo studies are extrapolated to reflect bioavailability of the drug formulation.

\section{Thermodynamic Stability and Surface Characteristics}

Although the physical appearance of a nanoemulsion may resemble that of a microemulsion, in that both systems may be transparent and of low viscosity, there is an essential difference between the two systems. A nanoemulsion is at best, kinetically stable, while microemulsion is thermodynamically stable [64]. Nanoemulsions because of their small droplet size, possess higher stability against sedimentation or creaming than microemulsions [65]. The two systems are very different since nanoemulsions are formed by mechanical shear and microemulsion phases are formed by self-assembly.

\section{Formulation of Nanoemulsions}

\subsection{Screening for Excipients Solubility}

The solubility of the drug in various oils, surfactants and cosurfactants is determined by dissolving an excess amount of the drug in small quantities of the selected oils, surfactants and cosurfactants and mixed using a mixer. A combination of oils can also be used for the determination of solubility.

The mixtures are allowed to equilibrate at ambient temperature in an isothermal shaker [66]. Samples are removed from the shaker and centrifuged. The supernatant is filtered through a $0.45 \mu \mathrm{m}$ membrane filter. The concentration of the drug is determined in each oil, surfactant, cosurfactant and combination of oils by HPLC or UV Spectrophotometer at their respective $\lambda_{\max }$.

\subsection{Construction of Pseudoternary Phase Diagram}

In order to find out the concentration range of components for the nanoemulsions, pseudo-ternary phase diagrams are constructed using water titration method at ambient temperature [67]. Different phase diagrams are prepared with varying weight ratios of surfactant to cosurfactant. These ratios are chosen in increasing concentration of surfactant with respect to cosurfactant and increasing concentration of cosurfactant with respect to surfactant for a detailed study of the phase diagrams. For each phase diagram at a specific surfactant: cosurfactant weight ratio, the ratios of oil to the mixture of surfactant and cosurfactant are varied. The mixtures of oil, surfactant and co-surfactant at certain weight ratios are diluted with water drop-wise, under moderate magnetic stirring. Visual observations are made for transparent and easily flowable nanoemulsions. The physical state of the nanoemulsions are marked on a pseudoternary phase diagram with one axis representing the aqueous phase, the second one representing oil and the third representing a mixture of surfactant and cosurfactant at a fixed weight ratio [66].

\subsection{Stability of Nanoemulsions}

Stability of a dosage form refers to the chemical and physical integrity of the dosage unit and when appropriate, the ability of the dosage unit to maintain protection against microbiological contamination $[68,69]$. Stability of drug product is one of the problems associated with the development of emulsions, microemulsions and nanoemulsions. Nanoemulsions have been known to enhance the physical as well as chemical stability of drugs [64,70,71].

Stability studies are performed on nanoemulsions by storing them at refrigerator and room temperatures over a number of months. The viscosity, refractive index and droplet size are determined during this period of storage. Insignificant changes in these parameters indicate formulation stability.

Accelerated stability studies can also performed. In 
this instance, nanoemulsion formulation are kept at accelerated temperatures and samples withdrawn at regular intervals and analyzed for drug content by stability indicating HPLC methods [69]. The amount of drug degraded and remaining in nanoemulsion formulation is determined at each time interval.

\section{Patents on Nanoemulsions}

Though many of them have not reached the market yet, a good number of patents have been received on nanoemulsion formulations. Probably due to the challenges of industrial scale production of nanoemulsions, few patents have been transferred into commercial products. Some patents related to nanoemulsions are presented in Table 1 [72-74].

\section{Commercial Nanoemulsions}

In spite of some difficulties, certain nanoemulsion formulations have been translated into commercial products, available in the market for use. Some commercial nanoemulsion formulations are listed in Table 2 [1].

\section{Nanoemulsion Formulation of Phytopharmaceuticals}

Phytopharmaceuticals have been formulated into microemulsions. The same cannot be said of nanoemulsions. Literature search showed few documented phytopharmaceutical microemulsions with different degrees of activity. A new self-microemulsifying drug delivery system has been successfully developed to improve the solubility

Table 1. Patents on nanoemulsion formulations.

\begin{tabular}{|c|c|c|}
\hline Patent Claim & Assignee & Patent Number \\
\hline $\begin{array}{l}\text { Transparent nanoemulsion less than } 100 \mathrm{~nm} \text { based on fluid } \\
\text { non-ionic amphiphilic lipids and use in cosmetics or in } \\
\text { dermopharmaceuticals }\end{array}$ & L'Oreal (Paris, FR) & US Patent number: $5,753,241$ \\
\hline $\begin{array}{l}\text { Nanoemulsions based on sugar fatty ethers and its uses in } \\
\text { the cosmetics, dermatological and/ophthalmological fields }\end{array}$ & L'Oreal (Paris, FR) & US Patent number: $6,689,371$ \\
\hline Method of preventing and treating microbial infections & NanoBio Corporation US & Patent Number: $6,506,803$ \\
\hline Nanoemulsion of 5-aminolevulinic acid & $\begin{array}{l}\text { ASAT AG Applied Science and Technology } \\
\qquad(\mathrm{Zug}, \mathrm{CH})\end{array}$ & PCT/EP99/08711 \\
\hline $\begin{array}{l}\text { Nanoemulsion of poorly soluble pharmaceutical active } \\
\text { ingredients and methods of making the same }\end{array}$ & & $\mathrm{WO} / 2007 / 103294$ \\
\hline $\begin{array}{l}\text { Nanoemulsion based on ethylene oxide \& propylene oxide } \\
\text { block copolymers and its use in the cosmetics, derma- } \\
\text { tological \& ophthalmological fields }\end{array}$ & L’Oreal (Paris, FR) & Patent Number: $6,464,990$ \\
\hline $\begin{array}{l}\text { Nanoemulsion based on glycerol fatty esters and its uses in } \\
\text { cosmetics, dermatological \& ophthalmological fields }\end{array}$ & L'Oreal (Paris, FR) & Patent Number: $6,541,018$ \\
\hline $\begin{array}{l}\text { Nanoemulsions based on oxyethylenated or non-oxyethy- } \\
\text { lenated sorbitan fatty esters and its uses in cosmetics, der- } \\
\text { matological and ophthalmological fields }\end{array}$ & L'Oreal (Paris, FR) & Patent Number: $6,335,022$ \\
\hline $\begin{array}{l}\text { Nanoemulsions based on phosphoric acid fatty acid esters } \\
\text { and its uses in cosmetics, dermatological and/ ophthalmo- } \\
\text { logical fields }\end{array}$ & L’Oreal (Paris, FR) & Patent Number: $6,274,150$ \\
\hline
\end{tabular}

Table 2. Commercial nanoemulsion formulations.

\begin{tabular}{ccc}
\hline Drug/Bioactive & Brand Name & Manufacturer \\
Palmitate alprostadil & Liple & Mitsubishi Pharmaceutical, Japan \\
Dexamethason & Limethason & Mitsubishi Pharmaceutical, Japan \\
Propofol & Diprivan & Astra Zaneca \\
Flurbiprofenaxtil & Ropion & Steroid \\
Vitamins A, D, E and $\mathrm{K}$ & Vitalipid & Anaesthetic \\
\hline
\end{tabular}


and oral absorption of curcumin [75]. Hesperetin, a flavonoid with anti-inflammatory, UV-protecting and antioxidant effect formulated into a microemulsion showed enhanced in vitro permeation compared to the aqueous and isopropyl myristate (IPM) suspension of hesperetin [76]. Neem oil microemulsion has also been formulated and its acaricidal activity investigated [77]. Since it is a function of globule size, these microemulsions could also be transformed to nanoemulsions through appropriate technologies that could cause breakdown of the globules to nano range.

\section{Major Challenges of Nanoemulsion Drug Delivery Systems}

Production of nanoemulsions requires significant energy input and although low-energy methods exist, they are not for industrial scale manufacture, low energy methods usually require high concentrations of surfactants and generally do not yield stable nanoemulsions.

Nanoemulsions are produced on industrial scale via the high-energy method which utilizes mechanical devices such as high pressure homogenizers which are very costly, extremely energy intensive and difficult to service. This challenge clearly accounts for the low translation of patented nanoemulsion formulations into commercial products.

There is also the lack of understanding of the mechanism of production of submicron droplets and the role of surfactants and cosurfactants as well as a lack of understanding of the interfacial chemistry that is involved in production of nanoemulsions [13]. For example, few formulation chemists are aware of the phase inversion temperature (PIT) concept and how this can be usefully applied for the production of small emulsion droplets.

Finally, there is the fear of introduction of new systems without full evaluation of the cost and benefits [13].

\section{Future Industrial Perspectives}

Nanoemulsion since its emergence has proved to be versatile and useful novel drug delivery system. Nanoemulsions are proposed for numerous applications in pharmacy as drug delivery systems because of their capacity of solubilizing non-polar active compounds. Future perspectives of nanoemulsion are very promising in different fields of therapeutics or application in development of cosmetics for hair or skin. One of the versatile applications of nanoemulsions is in the area of drug delivery where they act as efficient carriers for bioactives, facilitating administration by various routes. Their parenteral delivery has been adopted for supplying nutriational requirements, controlled drug release, vaccine delivery and for drug targeting to specific sites. The advantages and applications of oral drug delivery through these vehicles are numerous where the droplet size is related to their absorption in the gastrointestinal tract. Nanoemulsions have also been studied for their use in ocular delivery where pharmacological drugs are more sustained compared to their respective solutions. Pulmonary and transdermal routes are other successful ways of administering nanoemulsified delivery system. Although there have not been many reports of nanoemulsion applications in other fields, there is a great potential for nanoemulsion applications in other areas, such as in chemical and physical sciences, agriculture and engineering.

In the production of nanoemulsions there are some limitations, but pharmaceutical and food industries have to adjust their technologies to accommodate nanoemulsion production. Considering the versatile platforms nanoemulsions offer to formulation scientists in many fields, retooling of production facilities or outright change in technology of industries originally involved in production of parenteral and macro emulsions will lead to a lot economic windfall on the long run. This is because the effect of difficulty in preparation and the high energy input that may be involved in the production of nanoemulsion may just be felt on the short run. In as much as the cost of acquiring the technology for nanoemulsion production may be high, the production of nanoemulsions involves only a few steps, compensating the many steps involved in the production of some other products of lower versatility. Due to the renewed interest in herbal drug formulation, nanoemulsion may be the ideal delivery platform for these difficult-to-formulate phytopharmaceuticals. Novel nanoemulsion dosage forms of herbal drugs will lead to higher remuneration for the pharmaceutical industries.

With the advent of new instruments for high pressure homogenization and the competition between various manufacturers, the cost of production of nanoemulsions will decrease. Fundamental research in investigation of the role of surfactants in nanoemulsion production process will lead to optimized emulsifier systems and more economic use of surfactants will emerge. Nanoemulsions can be manipulated for targeted delivery and this hold significant promise in the area of oncology for the treatment of tumors and drug delivery to the brain.

\section{Conclusions}

The importance of design and development of emulsion nanocarrier systems aimed at controlling and/or improving required bioavailability levels of therapeutic agents cannot be overemphasized. Reducing droplet sizes to the nanoscale leads to some very interesting physical properties, such as optical transparency and unusual elastic behaviour. In the world of nanomaterials, nanoemulsions hold great promise as useful dispersions of deformable 
nanoscale droplets that can have different flow properties and optical properties ranging from opaque to nearly transparent. Moreover, it is very likely that nanoemulsions will play an increasingly important role comercially, since they can typically be formulated using significantly less surfactant than is required for nanostructured lyotropic microemulsion phases. The article has highlighted developments in this area. The various nanoemulsion carrier formulations developed so far have been identified. Nanoemulsions offer several advantages for the delivery of drugs and are thus receiving increasing attention as drug carriers for improving the delivery of active pharmaceutical ingredients. They are applicable for almost all routes of delivery and therefore hold promise for different fields, be it cosmetics, therapeutics or biotechnology.

\section{Expert Opinion}

Nanoemulsion drug delivery systems can be prepared by simple technology. They offer efficient targeting and controlled drug delivery. They also offer efficient protection of the encapsulated bioactive materials and enhanced delivery relative to most conventional dosage forms. The prospects lie in the ingenuity of formulation experts to utilize the advantages of nanoemulsion carriers in overcoming peculiar problems of drug delivery such as permeation and in vivo stability. We recommend more research efforts to exploit the potentials of emulsion nanotechnology in drug delivery of small molecule drugs and novel phytopharmaceuticals. This new technology could be developed to overcome the poor absorption of flavonoid nutrients and poor miscibility of these compounds with the lipid contents of cell membrane linings. The poor lipophilic affinity severely limits their ability to cross the lipid-rich enterocyte membrane.

\section{REFERENCES}

[1] P. Shah, D. Bhalodia and P. Shelat, "Nanoemulsion: A Pharmaceutical Review," Systematic Reviews in Pharmacy, Vol. 1, No. 1, 2010, pp. 24-32. doi:10.4103/0975-8453.59509

[2] T. P. U. Ravi and T. Padma, "Nanoemulsions for Drug Delivery through Different Routes," Research in Biotechnology, Vol. 2, No. 3, 2011, pp. 1-13.

[3] N. Anton and T. Vandamme, "The Universality of LowEnergy Nano-Emulsification," International Journal Pharmaceutics, Vol. 377, No. 1-2, 2009, pp. 142-147. doi:10.1016/j.ijpharm.2009.05.014

[4] T. G. Mason, S. M. Graves, J. N. Wilking and M. Y. Lin, "Extreme Emulsification: Formation and Structure of Nanoemulsions," Journal of Physics and Condensed Matter, Vol. 9, No. 1, 2006, pp. 193-199.

[5] S. Graves, K. Meleson and J. Wilking, "Structure of Concentrated Nanoemulsions," Journal of Chemical
Physics, Vol. 122, No. 13, 2005, Article ID 134703. doi:10.1063/1.1874952

[6] S. M. Jafari, Y. He and B. Bhandari, "Optimization of Nanoemulsion Production by Microfluidization," European Food Research Technology, Vol. 225, No. 5-6, 2007, pp. 733-741. doi:10.1007/s00217-006-0476-9

[7] C. Quin and D. J. Mc Clement, "Formation of Nanoemulsions Stabilized by Model Food Grade Emulsifiers Using High Pressure Homogenization: Factors Effecting Particle Size," Food Hydrocolloids, Vol. 25, No. 5, 2011, pp. 1000-1008. doi:10.1016/j.foodhyd.2010.09.017

[8] O. Sonneville-Aubrun, J. T. Simonnet and F. L'Alloret, "Nanoemulsions: A New Vehicle for Skincare Products," Advances in Colloids and Interface Science, Vol. 108-109, 2004, pp. 145-149. doi:10.1016/j.cis.2003.10.026

[9] C. Solans, P. Izquierdo, J. Nolla, N. Azemar and M. J. Garcia-Celma, "Nano-Emulsions," Current Opinion in Colloid and Interface Science, Vol. 10, No. 3-4, 2005, pp. 102-110. doi:10.1016/j.cocis.2005.06.004

[10] L. Wang, X. Li, G. Zhang, J. Dong and J. Eastoe, "Oil-in-Water Nanoemulsions for Pesticide Formulations," Journal of Colloid and Interface Science, Vol. 314, No. 1, 2007, pp. 230-235. doi:10.1016/j.jcis.2007.04.079

[11] I. Sole, A. Maestro, C. M. Pey, C. Gonzalez, C. Solans and J. M. Gutierrez, "Nanoemulsions Preparation by Low Energy Methods in an Ionic Surfactant System," Colloids and Surfaces A: Physiochemical Engineering Aspects, Vol. 288, 2006, pp. 138-143.

[12] I. Sole, C. M. Pey, A. Maestro, C. Gonzalez, M. Porras, C. Solans and J. M. Gutierrez, "Nanoemulsions Prepared by Phase Inversion Composition Method: Preparation Variables and Scale up," Journal of Colloid and Interface Science, Vol. 344, No. 2, 2010, pp. 417-423. doi:10.1016/j.jcis.2009.11.046

[13] T. Tadros, P. Izquierdo, J. Esquena and C. Solans, "Formation and Stability of Nanoemulsions," Advances in Colloids and Interface Science, Vol. 108-109, 2004, pp. 303-318. doi:10.1016/j.cis.2003.10.023

[14] R. Aboofazeli, "Nanometric Scaled Emulsions (Nanoemulsions)," Iranian Journal of Pharmaceutical Research, Vol. 9, No. 4, 2010, pp. 325-326.

[15] A. Pires, A. Fortuna, G. Alves and A. Falcao, "Intranasal Drug Delivery: How, Why and What for," Journal of Pharmacy and Pharmaceutical Sciences, Vol. 12, 2009, pp. 288-311.

[16] M. I. Ugwoke, R. U. Agu, N. Verbeke and R. Kinget, "Nasal Mucoadhesive Drug Delivery: Background, Applications, Trends, and Future Perspectives," Advanced Drug Delivery Reviews, Vol. 57, No. 11, 2005, pp. 16401665. doi:10.1016/j.addr.2005.07.009

[17] W. M. Pardridge, "Non-Invasive Drug Delivery to Human Brain Using Endogenous Blood Brain Barrier Transport System," Pharmaceutical Science and Technology Today, Vol. 2, No. 2, 1999, pp. 49-59. doi:10.1016/S1461-5347(98)00117-5

[18] M. Kumar, A. Misra, A. K. Babbar and A. K. Mishra, 
"Intranasal Nanoemulsion Based Brain Targeting Drug Delivery System of Risperidone," International Journal of Pharmaceutics, Vol. 358, 1-2, 2008, pp. 285-291. doi:10.1016/j.ijpharm.2008.03.029

[19] A. Mistry, S. Stolnik and L. Illum, "Nanoparticles for Direct Nose-to-Brain Delivery of Drugs," International Journal of Pharmaceutics, Vol. 379, No. 1, 2009, pp. 146-157. doi:10.1016/j.ijpharm.2009.06.019

[20] N. Csaba, M. Garcia-Fuentes and M. J. Alonsa, "Nanoparticles for Nasal Vaccination," Advanced Drug Delivery Reviews, Vol. 61, No. 2, 2009, pp. 140-157. doi:10.1016/j.addr.2008.09.005

[21] A. M. Clark, M. A. Jepson and B. H. Hirst, "Exploiting M Cells for Drug and Vaccine Delivery," Advanced Drug Delivery Reviews, Vol. 50, No. 1-2, 2001, pp. 81-106. doi:10.1016/S0169-409X(01)00149-1

[22] C. C. Muller-Goymann, "Physicochemical Characterization of Colloidal Drug Delivery Systems such as Reverse Micelles, Vesicles, Liquid Crystals and Nanoparticles for Topical Administration," European Journal of Pharmaceutics and Biopharmaceutics, Vol. 58, No. 2, 2004, pp. 343-356. doi:10.1016/j.ejpb.2004.03.028

[23] P. K. Gaur, S. Mishra, S. Purohit and K. Dave, "Transdermal Drug Delivery System: A Review," Asian Journal of Pharmaceutical and Clinical Research, Vol. 2, No. 1, 2009, pp. 14-20.

[24] F. Shakee and W. Ramadan, "Transdermal Delivery of Anticancer Drug Caffeine from Water-in-Oil Nanoemulsions," Colloids and Surfaces B: Biointerfaces, Vol. 75, No. 1, 2010, pp. 356-362. doi:10.1016/j.colsurfb.2009.09.010

[25] D. W. Osborne, A. J. Ward and K. J. Neil, "Microemulsions as Topical Delivery Vehicles: In-Vitro Transdermal Studies of a Model Hydrophilic Drug," Journal of Pharmacy and Pharmacology, Vol. 43, No. 6, 1991, pp. 450454. doi:10.1111/j.2042-7158.1991.tb03511.x

[26] M. Trotta, F. Pattarino and M. R. Gasco, "Influence of Counter Ions on the Skin Permeation of Methotrexate from Water-Oil Microemulsions," Pharmaceutia Acta Helvetiae, Vol. 71, No. 2, 1996, pp. 135-140. doi:10.1016/0031-6865(96)00003-9

[27] M. B. Delgado-Charro, G. Iglesias-Vilas, J. BlancoMendez, M. J. Lopez-Quintela, M. A. Marty and J. P. Guy, "Delivery of a Hydrophilic Solute through the Skin from Novel Microemulsion Systems," European Journal of Pharmaceutics and Biopharmaceutics, Vol. 43, No. 1, 1997, pp. 37-42. doi:10.1016/S0939-6411(96)00016-1

[28] F. Dreher, P. Walde, P. Walter and E. Wehrli, "Interaction of a Lecithin Microemulsion Gel with Human Stratum Corneum and Its Effect on Transdermal Transport," Journal of Controlled Release, Vol. 45, No. 2, 1997, pp. 131-140. doi:10.1016/S0168-3659(96)01559-3

[29] U. Schmalfus, R. Neubart and W. Wohlrab, "Modification of Drug Penetration into Human Skin Using Microemulsions," Journal of Controlled Release, Vol. 46, No. 3, 1997, pp. 279-285. doi:10.1016/S0168-3659(96)01609-4
[30] M. Kreilgaard, E. J. Pedersen and J. W. Jaroszewski, "NMR Characterization and Transdermal Drug Delivery Potentials of Microemulsion Systems," Journal of Controlled Release, Vol. 69, No. 3, 2000, pp. 421-433. doi:10.1016/S0168-3659(00)00325-4

[31] M. J. Alvarez-Figueroa and J. Blanco-Mendez, "Transdermal Delivery of Methotrexate: Iontophoretic Delivery from Hydrogels and Passive Delivery from Microemulsions," International Journal of Pharmaceutics, Vol. 215, No. 1-2, 2001, pp. 57-65.

[32] Y. S. Rhee, J. G. Choi, E. S. Park and S. C. Chi, "Transdermal Delivery of Ketoprofen Using Microemulsions," International Journal of Pharmaceutics, Vol. 228, No. 1, 2001, pp. 161-170.

[33] P. J. Lee, R. Langer and V. P. Shastri, "Novel Microemulsion Enhancer Formulation for Simultaneous Transdermal Delivery of Hydrophilic and Hydrophobic Drugs," Pharmaceutical Research, Vol. 20, No. 2, 2003, pp. 264-269. doi:10.1023/A:1022283423116

[34] J. Kemken, A. Ziegler and B. W. Muller, "Influence of Supersaturation on the Pharmacodynamic Effect of $\mathrm{Bu}-$ pranolol after Dermal Administration Using Microemulsions as Vehicle," Pharmaceutical Research, Vol. 9, No. 4, 1992, pp. 554-558. doi:10.1023/A:1015856800653

[35] M. Kreilgaard, "Dermal Pharmacokinetics of Microemulsion Formulations Determined by In-Vitro Microdialysis," Pharmaceutical Research, Vol. 18, No. 3, 2001, pp. 367-373. doi:10.1023/A:1011067300397

[36] M. Kreilgaard, M. J. B. Kemme, J. Burggraaf, R. C. Schoemaker and A. F. Cohen, "Influence of a Microemulsion Vehicle on Cutaneous Bioequivalence of a Lipophilic Model Drug Assessed by Microdialysis and Pharmacodynamics," Pharmaceutical Research, Vol. 18, No. 5, 2001, pp. 593-599. doi:10.1023/A:1011068907416

[37] G. Ktistis and I. Niopas, "A Study on the In-Vitro Percutaneous Absorption of Propranolol from Disperse Systems," Journal of Pharmacy and Pharmacology, Vol. 50, 1998, pp. 413-419. doi:10.1111/j.2042-7158.1998.tb06881.x

[38] M. R. Gasco, M. Gallarate and F. Pattarino, "In Vitro Permeation of Azelaic Acid from Viscosized Microemulsions," International Journal of Pharmaceutics, Vol. 69. 1999, pp. 193-196.

[39] K. Kriwet and C. C. Muller-Goymann, "Diclofenac Release from Phospholipid Drug Systems and Permeation through Excised Human Stratum Corneum," International Journal of Pharmaceutics, Vol. 125, No. 2, 1995, pp. 231-242. doi:10.1016/0378-5173(95)00130-B

[40] M. Trotta, "Influence of Phase Transformation on Indomethacin Release from Microemulsions," Journal of Controlled Release, Vol. 60, No. 2, 1999, pp. 399-405. doi:10.1016/S0168-3659(99)00094-2

[41] R. Patel and K. P. Patel, "Advances in Novel Parenteral Drug Delivery Systems," Asian Journal of Pharmaceutics, Vol. 4, No. 3, 2010, pp. 193-199. doi: $10.4103 / 0973-8398.72117$ 
[42] F. A. Araujo, R. G. Kelmann, B. V. Araujo, R. B. Finatto, H. F. Teixeira and L. S. Koester, "Development and Characterization of Parenteral Nanoemulsions Containing Thalidomide," European Journal of Pharmaceutical Science, Vol. 42, No. 5, 2011, pp. 238-245. doi:10.1016/j.ejps.2010.11.014

[43] S. Ganta, D. Deshpande, A. Korde and M. Amiji, "A Review of Multifunctional Nanoemulsion Systems to Overcome Oral and CNS Drug Delivery Barriers," Molecules and Membrane Bioliology, Vol. 27, No. 7, 2010, pp. 260-273. doi:10.3109/09687688.2010.497971

[44] R. G. Kelmann, G. Kuminek, H. Teixeira and L. S. Koester, "Carbamazepine Parenteral Nanoemulsions Prepared by Spontaneous Emulsification Process," International Journal of Pharmaceutics, Vol. 342, No. 1-2, 2007, pp. 231-239. doi:10.1016/j.ijpharm.2007.05.004

[45] F. L. Primo, L. Michieloto, M. A. M. Rodrigues, P. P. Macaroff, P. C. Morais, Z. G. M. Lacava, M. V. L. B. Bently and A. C. Tedesco, "Magnetic Nanoemulsions as Drug Delivery System for Foscan: Skin Permeation and Retention in Vitro Assays for Topical Application in Photodynamic Therapy (PDT) of Skin Cancer," Journal of Magnetism and Magnetic Materials, Vol. 311, No. 1, 2007, pp. 354-357. doi:10.1016/j.jmmm.2006.10.1183

[46] A. Bielinska, K. Janxzak and J. Landers, "Nasal Immunization with a Recombinant HIV gp120 and Nanoemulsion Adjuvant Produces Thl polarized Responses and Neutralizing Antibodies to Primary HIV Type Isolates," AIDS Research and Human Retroviruses, Vol. 24, No. 2, 2008, pp. 271-281. doi:10.1089/aid.2007.0148

[47] A. C. Berkowltz and D. M. Goddard, "Novel Drug Delivery Systems: Future Directions," Pharmacology Update, April 2009.

[48] H. M. Mansour, Y. S. Rhee and W. Xiao, "Nanomedicine in Pulmonary Delivery," International Journal of Nanomedicine, Vol. 4, 2009, pp. 299-319. doi:10.2147/IJN.S4937

[49] F. Liu, J. Yang, L. Huang and D. Liu, "Effect of NonIonic Surfactants on the Formation of DNA/Emulsion Complexes and Emulsion-Mediated Gene Transfer," Pharmaceutical Research, Vol. 13, No. 11, 1996, pp. 1642-1646. doi:10.1023/A:1016480421204

[50] S. W. Yi, T. Y. Yune, T. W. Kim, H. Chung, et al., "A Cationic Lipid Emulsion/DNA Complex as a Physically Stable and Serum-Resistant Gene Delivery System," Pharmaceutical Research, Vol. 17, No. 3, 2000, pp. 314-320. doi:10.1023/A:1007553106681

[51] C.-H. Liu and S.-Y. Yu, "Cationic Nanoemulsions as Non-Viral Vectors for Plasmid DNA Delivery," Colloids and Surfaces B: Biointerfaces, Vol. 79, No. 2, 2010, pp. 509-515. doi:10.1016/i.colsurfb.2010.05.026

[52] M. Bivas-Benita, M. Oudshoorn, S. Romeijn, et al., "Cationic Submicron Emulsions for Pulmonary DNA Immunization," Journal of Control Release, Vol. 100, No. 1, 2004, pp. 145-155. doi:10.1016/j.jconrel.2004.08.008

[53] http://www.usmedicine.com

[54] J. B. Kayes, "Disperse Systems," In: M. E. Aulton, Ed.,
Pharmaceutics the Science of Dosage Form Design, 1st Edition, Churchill Livingstone, Edinburgh, 1999, pp. 81-118, 571.

[55] M. J. Barea, M. J. Jekins, M. H. Gaber, et al., "Evaluation of Liposomes Coated with a $\mathrm{pH}$ Responsive Polymer," International Journal of Pharmaceutics, Vol. 402, No. 1, 2010, pp. 89-94.

[56] N. A, Samah, N. Williams and C. M. Heard, "Nanogel Particulates Located within Diffusion Cell Receptor Phases Following Topical Application Demonstrates Uptake into and Migration Across Skin," International Journal of Pharmaceutics, Vol. 401, No. 1-2, 2010, pp. 72-78. doi:10.1016/j.ijpharm.2010.08.011

[57] H. S. Ruth, D. Attwood, G. Ktistis and C. Taylor, "Phase Studies and Particle Size Analysis of Oil-in-Water Phospholipid Microemulsions," International Journal of Pharmaceutics, Vol. 116, No. 2, 1995, pp. 253-261. doi:10.1016/0378-5173(94)00316-W

[58] X. Li, N. Anton, T. M. C. Ta, M. Zhao, N. Messaddeq and T. F. Vandamme, "Microencapsulation of Nanoemulsions: Novel Trojan Particles for Bioactive Lipid Molecule Delivery," International Journal of Nanomedicine, Vol. 2011, No. 6, 2011, pp. 1313-1325.

[59] Y. Agrawal, K. C. Petkar and K. K. Sawant, "Development, Evaluation and Clinical Studies of Acitretin Loaded Nanostructured Lipid Carriers for Topical Treatment of Psoriasis," International Journal of Pharmaceutics, Vol. 401, No. 1-2, 2010, pp. 93-102. doi:10.1016/j.ijpharm.2010.09.007

[60] E. Touitou, N. Dayan, L. Bergelson, et al., "EthosomesNovel Vesicular Carriers for Enhanced Delivery: Characterization and Skin Penetration Properties," Journal of Controlled Release, Vol. 65, No. 3, 2000, pp. 403-418. doi:10.1016/S0168-3659(99)00222-9

[61] E. R. Bendas and M. I. Tadros, "Enhanced Transdermal Delivery of Salbutamol Sulfate via Ethosomes," AAPS PharmSciTech, Vol. 8, No. 4, 2007, pp. 214-220.

[62] S. Jain, A. K. Tiwary, B. Sapra, et al., "Formulation and Evaluation of Ethosomes for Transdermal Delivery of Lamivudine," AAPS PharmSciTech, Vol. 8, No. 4, 2007, pp. 249-257. doi:10.1208/pt0804111

[63] V. Dave, D. Kumar, S. Lewis, et al., "Ethosome for Enhanced Transdermal Drug Delivery of Aceclofenac," International Journal of Drug Delivery, Vol. 2, 2010, pp. 81-92. doi:10.5138/ijdd.2010.0975.0215.02016

[64] S. Shafiq, S. Faiyaz, T. Sushma, F. J. Ahmad, R. K. Khar and M. Ali, "Development and Bioavailability Assessment of Ramipril Nanoemulsion Formulation," European Journal of Pharmaceutics and Biopharmaceutics, Vol. 66, No. 2, 2007, pp. 227-243.

doi:10.1016/j.ejpb.2006.10.014

[65] S. Amselem and D. Friedman, "Submicron Emulsions as Drug Carriers for Topical Administration," In: S. Benita, Ed., Submicron Emulsions in Drug Targeting and Delivery, The Netherlands, Harwood Academic Publishers, Amsterdam, 1998, pp. 153-173.

[66] F. Shakeel, S. Baboota, A. Ahuja, J. Ali, M. Aqil and S. 
Shafiq, "Nanoemulsions as Vehicles for Transdermal Delivery of Aceclofenac," AAPS PharmSciTech, Vol. 8, No. 4, 2007, pp. 191-199. doi:10.1208/pt0804104

[67] K. G. Pradip, J. Rita, M. U. Majithya and S. R. Rayassa, "Design and Development of Microemulsion Drug Delivery System of Acyclovir for Improvement of Oral Bioavailability," AAPS PharmSciTech, Vol. 7, No. 3, 2006, pp. 1-6.

[68] A. G. Floyd, "Top Ten Considerations in the Development of Parenteral Emulsions," Pharmaceutical Science and Technology Today, Vol. 2, No. 4, 1999, pp. 134-143. doi:10.1016/S1461-5347(99)00141-8

[69] F. Shakeel, S. Baboota, A. Ahuja, J. Ali, M. S Faisal and S. Shafiq, "Stability Evaluation of Celecoxib NanoemulSion Containing Tween 80," Thailand Journal of Pharmaceutical Science, Vol. 32, 2008, pp. 4-9.

[70] F. Shakeel, S. Baboota, A. Ahuja, J. Ali and S. Shafiq, "Skin Permeation Mechanism of Aceclofenac Using Novel Nanoemulsion Formulation," Pharmazie, Vol. 63, No. 8, 2008, pp. 580-584.

[71] S. Baboota, F. Shakeel, A. Ahuja, J. Ali and S. Shafiq, "Design Development and Evaluation of Novel Nanoe- mulsions Formulations for Transdermal Potential of Celecoxib," Acta Pharmaceutica, Vol. 8, 2007, pp. 316332.

[72] http://www.pharmacast.com

[73] http://www.wipo.org

[74] http://patentstorm.us

[75] J. Cui, B. Yu, W. Zhao, H. Li, H. Lou and G. Zhai, "Enhancement of Oral Absorption of Curcumin by Self-Microemulsifying Drug Delivery Systems," International Journal of Pharmaceutics, Vol. 371, No. 1-2, 2009, pp. 148-155. doi:10.1016/j.ijpharm.2008.12.009

[76] Y. Tsai, K. Lee, Y. Huang and P. Wu, "In Vitro Permeation and in Vivo Whitening Effect of Topical Hesperetin Microemulsion Delivery System," International Journal of Pharmaceutics, Vol. 388, No. 1-2, 2010, pp. 257-262. doi:10.1016/j.ijpharm.2009.12.051

[77] J. Xu, et al., "The Preparation of Neem Oil Microemulsion (Azadirachta indica) and the Comparison of Acaricidal Time between Neem Oil Microemulsion and Other Formulations in Vitro," Veterinary Parasitology, Vol. 169, No. 3-4, 2010, pp. 399-403. doi:10.1016/j.vetpar.2010.01.016 